\title{
ON MINIMAL SURFACES WITH THE RICCI CONDITION IN SPACE FORMS
}

\author{
By Makoto SaKaki
}

\section{Introduction}

A 2-dimensional Riemannian metric $d s^{2}$ is said to satisfy the Ricci condition with respect to $c$ if its Gaussian curvature $K$ satisfies $K<c$ and the new metric $d \hat{s}^{2}=$ $\sqrt{c-K} d s^{2}$ is flat.

Let $X^{N}(c)$ denote the $N$-dimensional simply connected space form of constant curvature $c$, and in particular, let $\mathbf{R}^{N}=X^{N}(0)$. The induced metric $d s^{2}$ on a minimal surface in $X^{3}(c)$ satisfies the Ricci condition with respect to $c$ except at points where the Gaussian curvature $=c$. Conversely, assume that a Riemannian metric $d s^{2}$ on a 2-dimensional simply connected manifold $M$ satisfies the Ricci condition with respect to $c$. Then there exists a smooth $2 \pi$-periodic family of isometric minimal immersions $f_{\theta}:\left(M, d s^{2}\right) \rightarrow X^{3}(c) ; \theta \in \mathbf{R}$, which is called the associated family. Moreover, up to congruences, the maps $f_{\theta} ; 0 \leqq \theta<\pi$ represent all local isometric minimal immersions of $\left(M, d s^{2}\right)$ into $X^{3}(c)$ (see [5]). So, the Ricci condition with respect to $c$ is an intrinsic characterization of minimal surfaces in $X^{\mathbf{3}}(c)$.

Here we consider the following problem, which may be seen as a kind of rigidity problem.

Problem. Classify those minimal surfaces in $X^{N}(c)$ whose induced metrics satısfy the Ricci condition with respect to $c$, or equivalently, classify those minımal surfaces in $X^{N}(c)$ which are locally isometric to minımal surfaces in $X^{3}(c)$.

A submanifold in $X^{N}(c)$ is said to lie fully in $X^{N}(c)$ if it does not lie in a totally geodesic submanifold of $X^{N}(c)$. Let $S(N, c)$ denote the set of all Riemannian structures of minimal surfaces lying fully in $X^{N}(c)$. Then the problem is to determine the intersection of $S(3, c)$ and $S(N, c)$.

\section{Examples}

In this section, we give examples of minimal surfaces in $X^{N}(c)$ which do not lie in a totally geodesic $X^{3}(c)$ and whose induced metrics satisfy the Ricci condition with respect to $c$. The following three types of examples are known.

Example $1([6])$. Let $f_{\theta}:\left(M, d s^{2}\right) \rightarrow \mathbf{R}^{3} ; \theta \in \mathbf{R}$ be the associated family of isometric minimal immersions of a 2-dimensional Riemannian manifold $\left(M, d s^{2}\right)$ into 
$\mathbf{R}^{3}$. Then we can construct an isometric minimal immersion $f:\left(M, d s^{2}\right) \rightarrow \mathbf{R}^{6}$ by setting

$$
f=f_{\theta} \cos \varphi \oplus f_{\theta+\pi / 2} \sin \varphi
$$

where the symbol $\oplus$ denotes the direct sum with respect to an orthogonal decomposition $\mathbf{R}^{6}=\mathbf{R}^{3} \oplus \mathbf{R}^{3}$. The metric induced by $f$ is $d s^{2}$, which satisfies the Ricci condition with respect to 0 except at points where the Gaussian curvature $=0$. Furthermore, in general, $f(M)$ lies fully in $\mathbf{R}^{6}$ if $\varphi \neq 0(\bmod \pi / 2)$.

Example $2([6])$. Let $c>0$. Let $f_{\theta}:\left(M, d s^{2}\right) \rightarrow X^{3}(c)\left(\subset \mathbf{R}^{4}\right) ; \theta \in \mathbf{R}$ be the associated family of isometric minimal immersions of a 2-dimensional Riemannian manifold $\left(M, d s^{2}\right)$ into $X^{3}(c)$. Then we can construct an isometric minimal immersion $f:\left(M, d s^{2}\right) \rightarrow X^{4 m+3}(c)\left(\subset \mathbf{R}^{4 m+4}\right)$ by setting

$$
f=a_{0} f_{\theta_{0}} \oplus \cdots \oplus a_{m} f_{\theta_{m}}
$$

where $\sum_{z=1}^{m} a_{i}^{2}=1,0 \leqq \theta_{0}<\theta_{1}<\cdots<\theta_{m}<\pi$, each $f_{\theta_{i}}$ is viewed as an $\mathbf{R}^{4}$-valued function with $\left|f_{\theta_{i}}\right|=1 / \sqrt{c}$, and the symbol $\oplus$ denotes the direct sum with respect to an orthogonal decomposition $\mathbf{R}^{4 m+4}=\mathbf{R}^{4} \oplus \cdots \oplus \mathbf{R}^{4}$. The metric induced by $f$ is $d s^{2}$, which satisfies the Ricci condition with respect to $c$ except at points where the Gaussian curvature $=c$. Furthermore, in general, $f(M)$ lies fully in $X^{4 m+3}(c)$.

Example 3 ([1] and [4]). Every 2-dimensional flat metric automatically satisfies the Ricci condition with respect to $c>0$, and there are flat minimal surfaces lying fully in $X^{2 n+1}(c)$ where $c>0$.

\section{Known results}

In the Euclidean case where $c=0$, Lawson solved the problem completely as follows.

Theorem 1 ([6] AND [7, Chapter IV]). Let $f: M \rightarrow \mathbf{R}^{N}$ be a minımal $\imath m-$ mersion of a 2-dimensional manifold $M$ into $\mathbf{R}^{N}$. Suppose that the induced metric ds ${ }^{2}$ satisfies the Ricci condition with respect to 0 except at isolated points where the Gaussian curvature $=0$. Then either (i) $f(M)$ lies in a totally geodesic $\mathbf{R}^{3}$, or (iv) $f(M)$ lies fully in a totally geodesıc $\mathbf{R}^{6}$ and $f$ is of the form of $(1)$ in Example 1 for $\varphi \neq 0(\bmod \pi / 2)$.

Remark 1. Theorem 1 says that $S(3,0)$ and $S(N, 0)$ are disjoint if $N=4, N=5$ or $N \geqq 7$. Theorem 1 says also that $S(3,0)$ is included in $S(6,0)$ through Example 1 .

Concerning the spherical case where $c>0$, Lawson posed the following conjecture.

Conjecture ([6]). Let $f: M \rightarrow X^{N}(c)$ be a minımal ımmersıon of a 2-dimensional manifold $M$ into $X^{N}(c)$ where $c>0$. Suppose that the induced metric ds ${ }^{2}$ satisfies the Ricci condition with respect to $c$ except at isolated points where the Gaussian curvature $=c$. Then $f$ must be of the form of (2) in Example 2.

As a matter of fact, there are easy counter-examples to this conjecture (cf. Example 3 ). So one should consider the conjecture for non-flat minimal surfaces. In [8], with some 
global assumptions, Naka (= Miyaoka) obtained partial positive answers to this question.

\section{Our results}

First we solve the problem in the case where $N=4$.

THEOREM $2([10])$. Let $f: M \rightarrow X^{4}(c)$ be a minımal ımmersion of a 2-dimensıonal manifold $M$ into $X^{4}(c)$. Suppose that the induced metric ds ${ }^{2}$ satisfies the Ricci condition with respect to $c$ except at isolated points where the Gaussian curvature $=c$. Then $f(M)$ lies in a totally geodesic $X^{3}(c)$.

Remark 2. (i) Theorem 2 says that $S(3, c)$ and $S(4, c)$ are disjoint.

(ii) When $c=0$, Theorem 2 is included in [6].

(iii) In the case where $c>0$, Theorem 2 is not true if we replace $X^{4}(c)$ by $X^{5}(c)$ (cf. Example 3).

(iv) In [10], with an additional assumption, we give a result also in higher codimensional cases.

In [3] Johnson studied a class of minimal surfaces in $X^{N}(c)$, which are called exceptional minimal surfaces and are related to the theory of harmonic sequences (cf. [2] and [11]). Next we discuss exceptional minimal surfaces in $X^{N}(c)$ whose induced metrics satisfy the Ricci condition with respect to $c$.

Theorem $3([9])$. Let $f: M \rightarrow X^{N}(c)$ be an exceptional minımal ımmersion of a 2-dimensional manifold $M$ into $X^{N}(c)$ where $c>0$. Suppose that the induced metric $d s^{2}$ satisfies the Ricci condition with respect to $c$ except at isolated points where the Gaussian curvature $=c$. Then either (i) $f(M)$ lies fully in a totally geodesics $X^{4 m+1}(c)$ and $d s^{2}$ is flat, or (ii) $f(M)$ lies fully in a totally geodesıc $X^{4 m+3}(c)$.

THEOREM $4([9])$. Let $f: M \rightarrow X^{N}(c)$ be an exceptional minımal ımmersion of a 2-dimensional manifold $M$ into $X^{N}(c)$ where $c<0$. Suppose that the induced metric ds ${ }^{2}$ satisfies the Ricci condition with respect to $c$ except at isolated points where the Gaussian curvature $=c$. Then $f(M)$ lies in a totally geodesic $X^{3}(c)$.

Remark 3. (i) There are flat exceptional minimal surfaces lying fully in $X^{2 n+1}(c)$, where $c>0$ (see [9]).

(ii) There are non-flat exceptional minimal surfaces lying fully in $X^{4 m+3}(c)$ whose induced metrics satisfy the Ricci condition with respect to $c$, where $c>0$ (see [9]).

\section{REFERENCE}

[1] R. Bryant, Minimal surfaces of constant curvature in $S^{n}$, Trans. Amer. Math. Soc. 290 (1985), 259-271.

[2] S.S. Chern and J.G. Wolfson, Harmonic maps of the two-sphere into a complex Grassmann manifold II, Ann. of Math. 125 (1987), 301-335. 
[ 3 ] G.D. Johnson, An intrinsic characterization of a class of minimal surfaces in constant curvature manifolds, Pacific J. Math. 149 (1991), 113-125.

[ 4 ] K. Kenmotsu, On minimal immersions of $\mathbf{R}^{2}$ into $S^{N}$, J. Math. Soc. Japan 28 (1976), 182-191.

[ 5 ] H.B. Lawson, Complete minimal surfaces in $S^{3}$, Ann. of Math. 92 (1970), 335-374.

[6] H.B. Lawson, Some intrinsic characterizations of minimal surfaces, J. Analyse Math. 24 (1971), 151-161.

[ 7 ] H.B. Lawson, Lectures on Minimal Submanifolds, Publish or Perish, Inc., Berkeley, 1980.

[8] R. Naka, Some results on minimal surfaces with the Ricci condition, Minimal Submanifolds and Geodesics (M. Obata, ed.), 1978, Kaigai Publ., Tokyo, 121-142.

[ 9 ] M. Sakaki, Exceptional minimal surfaces with the Ricci condition, Tsukuba J. Math. 16 (1992), 161-167.

[10] M. Sakaki, Minimal surfaces with the Ricci condition in 4-dimensional space forms, Proc. Amer. Math. Soc., 121 (1994), 573-577.

[11] J.G. Wolfson, Harmonic sequences and harmonic maps of surfaces into complex Grassmann manifolds, J. Diff. Geom. 27 (1988), 161-178.

Department of Mathematics

FACULTY OF SCIENCE

HIROSAKI UNIVERSITY

HIROSAKI 036, JAPAN 